www.jmscr.igmpublication.org

Impact Factor (SJIF): 6.379

Index Copernicus Value: 71.58

ISSN (e)-2347-176x ISSN (p) 2455-0450

crossref DOI: _https://dx.doi.org/10.18535/jmscr/v6i4.131

Journal Of Medical Science And Clinical Research

\title{
Antimicrobial resistance pattern of ESBLs producing uropathogenic E. coli (UPEC) in hospitalized patients from a tertiary care hospital of central India
}

\author{
Authors \\ Dr Arvind Khodare ${ }^{1}$, Dr Manish Purohit ${ }^{2}$, Dr Anita Mutha ${ }^{3}$ \\ ${ }^{1}$ Senior Resident, Dept of Microbiology, MGM Medical College, Indore \\ Mob No.8964011927, Email:1412abi@gmail.com \\ ${ }^{2}$ Associate Prof, Microbiology, MGM Medical College, Indore \\ ${ }^{3}$ Prof \& Head, Dept of Microbiology, MGM, Medical College, Indore \\ Mob: 9826170789, Email: dranitamutha@ gmail.com \\ Corresponding Author \\ Dr Manish Purohit \\ Associate Prof, Microbiology, MGM Medical College, Indore \\ Mob:8109671170, Email: manishpurohit75@yahoo.co.in
}

\begin{abstract}
Detection of ESBLs producing organisms from samples such as urine represents an epidemiologic marker of colonization. Thus the present study was aimed to know the antimicrobial resistance pattern among ESBL producing urinary isolates of E. coli which will helps in deciding empiric antibiotic therapy and identifying measures to reduce increasing resistance trends. 106 consecutive non repeating uropathogenic E. coli included in the study. All specimens were cultured using a calibrated loop. Identification of E. coli was done by standard biochemical tests. Antimicrobial susceptibility testing was done by Kirby-Bauer's disc diffusion method, isolates were screened for ESBLs production and confirmation by combination disk method as per CLSI 2014 guidelines. E. coli ATCC 25922 and Klebsiella pneumonia ATCC 700603 were used as a negative control and positive control respectively. Out of 106 uropathogenic E. Coli, 99(93.4\%) strains identified by ESBL screening and 48 (45.2\%) were confirmed for ESBLs production. Minimum resistance to Imipenem (1\%) followed by Meropenem (2\%) and Nitrofurantoin (4\%), and maximum resistance to Piperacillin(100\%) followed by Cefipime (96\%), Ciprofloxacin (96\%) and Norfloxacin (91\%) were seen. Thus Imipenem followed by Nitrofurantoin and Amikacin remains the drug of choice for ESBL producers with the highest sensitivity depending on severity and risk factors.
\end{abstract}

Keywords: HAI, UTI, beta-lactamase.

\section{Introduction}

Urinary tract infections (UTIs) are among the most common bacterial infections. ${ }^{1}$ E. Coli causes $70-90 \%$ cases of community-acquired UTIs, $85 \%$ cases of asymptomatic bacteriuria and more than $60 \%$ cases of recurrent cystitis. ${ }^{2}$ UTIs is also among one of the major hospital acquired infection, accounting for as many as $35 \%$ of nosocomial infections, and they are the second most common cause of bacteremia in hospitalized patients. 3,4

Antimicrobial resistance has been recognized as an emerging worldwide problem both in developed and developing countries. ${ }^{5}$ The effect 
could be severe in heavily populated developing country such as India where there is no strict monitoring program regarding the use of antibiotics. In Enterobacteriaceae antimicrobial resistance in $E$. coli is of particular concern because it is the most common Gram negative pathogen causing infections in human being, particularly urinary tract infections (UTIs). Antimicrobial drug resistance frequency is increasing worldwide with regional differences. ${ }^{6}$ In principle, any organism could develop resistance to any antibiotic. ${ }^{7}$ The extended spectrum $\beta$-Lactamase (ESBL) enzymes are plasmid-mediated enzymes capable of hydrolyzing and inactivating a wide variety of $\beta$ Lactams, including third generation Cephalosporins, Penicillins and Aztreonam. ${ }^{8}$ Due to the production of extended spectrum $\beta$ Lactamases (ESBLs) E. coli exhibits increasing resistance to $\beta$-Lactam antibiotics. Plasmids responsible for ESBL production carry resistance to many antibiotics like Aminoglycosides, Fluoroquinolones, Tetracyclines, Chloramphenicol and co-trimoxazole. ${ }^{9,10}$ Detection of ESBLs producing organisms from samples such as urine represents an epidemiologic marker of colonization, and therefore there is potential for transfer of such organisms to others. ${ }^{1}$ The present study was aimed to know the antimicrobial resistance pattern among ESBL producing urinary isolates of E. coli which will helps in deciding empiric antibiotic therapy and identifying measures to reduce increasing resistance trends.

\section{Material \& Methods}

106 consecutive non repeating uropathogenic $E$. coli included in the study. Strains were isolated from urine specimens of hospitalised patients (M.Y. Hospital, Indore) of different age groups with clinical symptoms of urinary tract infections which yielded significant growth on semi quantitative culture. The study period was of one year from July 2015 to June 2016.

All specimens were cultured on blood agar, MacConkey and UTI Crome agar plates using a calibrated loop (4mm diameter, Calibrated to $0.01 \mathrm{ml}$ ) and incubated aerobically at $37^{\circ} \mathrm{C}$. After overnight incubation, they were examined for growth of microorganisms. ${ }^{11}$ The E. coli were identified by their colony colour on UTI Crome Agar, colony morphology, staining characters, motility and other relevant biochemical tests (fermentation of lactose, ability to produce Indole, reaction on triple sugar Iron agar (TSI), citrate and urease utilization). ${ }^{11}$ Antimicrobial susceptibility testing was done by Kirby-Bauer's disc diffusion method as per the CLSI guidelines. ${ }^{8}$

\section{Screening test for ESBLs}

Isolates were screened for ESBLs production by using standard CLSI disc Diffusion method and criteria. Four disk of Cefotaxime (CTX), Ceftazidime (CAZ), ceftriaxone (CTR) and Cefpodoxime (CPD) were used for susceptibility testing by Kirby-Bauer's disk diffusion testing. Inhibition zone size of $\geq 22 \mathrm{~mm}$ for ceftazidime $(30 \mu \mathrm{g}), \geq 25 \mathrm{~mm}$ for ceftriaxone $(30 \mu \mathrm{g}), \geq 27 \mathrm{~mm}$ for cefotaxime $(30 \mu \mathrm{g})$ and $\geq 17 \mathrm{~mm}$ for cefpodoxime $(10 \mu \mathrm{g})$ indicate probable ESBL production. $^{8}$

\section{Phenotypic Confirmatory test for ESBLs production}

Phenotypic confirmation of ESBL were done by combination disk method as per CLSI 2014 guidelines. ${ }^{8}$ In this test a disc of Ceftazidime $(30 \mu \mathrm{g})$ and Cefotaxime $(30 \mu \mathrm{g})$ alone, and in combination with clavulanic acid $(10 \mu \mathrm{g})$ were used. A $\geq 5 \mathrm{~mm}$ increase in zone diameter when tested with clavulanic acid was designated as ESBL positive. ${ }^{8}$

E. coli ATCC 25922 and Klebsiella pneumonia ATCC 700603 were used as a negative control and positive control respectively.

\section{Results}

Out of 106 uropathogenic E. Coli, 99(93.4\%) strains identified by ESBL screening and 48 (45.2\%) were confirmed for ESBLs production. Multiple drug resistance were observed in ESBLs producing strains of E. coli. Minimum resistance 
to Imipenem (1\%) followed by Meropenem (2\%) and Nitrofurantoin (4\%), and maximum resistance to Piperacillin(100\%) followed by Cefipime
(96\%), Ciprofloxacin (96\%) and Norfloxacin (91\%) were seen.(Table 1 )

\begin{tabular}{|l|c|}
\hline Table : Antibiotic resistance pattern of ESBLs producing $\boldsymbol{E}$. coli, $\mathbf{n}=\mathbf{4 8}$ \\
\hline Imipenem $(10 \mu \mathrm{g})$ & $1(2 \%)$ \\
\hline Meropenem $(10 \mu \mathrm{g})$ & $2(4 \%)$ \\
\hline Nitrofurantoin $(300 \mu \mathrm{g})$ & $4(8 \%)$ \\
\hline Amikacin $(30 \mu \mathrm{g})$ & $6(12.5 \%)$ \\
\hline Piperacillin-tazobactam $(100 / 10 \mu \mathrm{g})$ & $13(27 \%)$ \\
\hline Gentamicin $(10 \mu \mathrm{g})$ & $19(40 \%)$ \\
\hline Tetracycline $(30 \mu \mathrm{g})$ & $33(69 \%)$ \\
\hline Amoxycillin-clavulanic acid $(20 / 10 \mu \mathrm{g})$ & $36(75 \%)$ \\
\hline Trimethoprim-sulphamethoxazole $(1.25 / 23.75 \mu \mathrm{g})$ & $40(83 \%)$ \\
\hline Levofloxacin $(5 \mu \mathrm{g})$ & $42(88 \%)$ \\
\hline Norfloxacin $(10 \mu \mathrm{g})$ & $43(90 \%)$ \\
\hline Cefipime $(30 \mu \mathrm{g})$ & $45(94 \%)$ \\
\hline Ciprofloxacin $(5 \mu \mathrm{g})$ & $45(94 \%)$ \\
\hline Piperacillin $(100 \mu \mathrm{g})$ & $48(100 \%)$ \\
\hline
\end{tabular}

Figure- Antibiotic resistance pattern

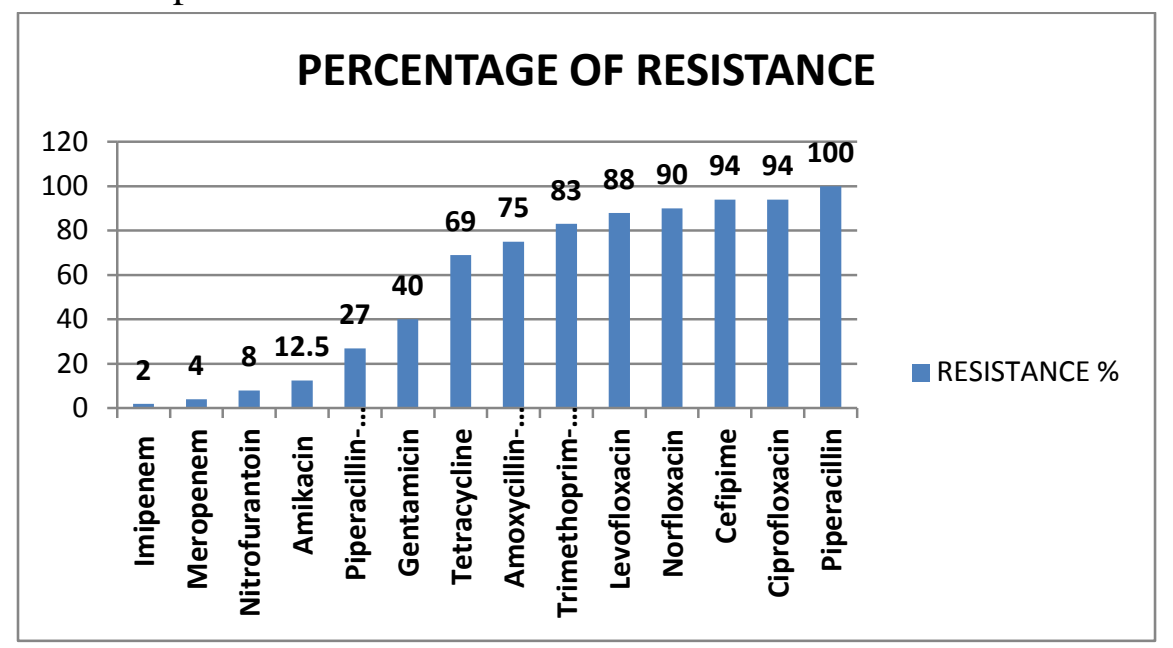

\section{Discussion}

Emergence and re-emergence of antimicrobial resistance shows the way to therapeutic failure especially of empirical therapy. The problem is further substantially enhanced throughout the world with the emergence of ESBL strains. For that reason, the information about the prevalence of local and surrounding pathogens and their antimicrobial susceptibility pattern are vital for clinicians to treat patients effectively. The prevalence of ESBLs producing organisms among clinical isolates vary greatly geographically and rapidly changing over time. Thus, it is worth it to state that throughout the India the prevalence of ESBL producers may vary profoundly and patterns change rapidly over time. The incidence of Urinary tract infections by ESBL producing $E$. coli was found highest in India (60\%) followed by Hongkong (48\%) and Singapore (33\%). ${ }^{12}$ In India, second generation Cephalosporins and even some third generation Cephalosporins are extensively used by general practitioners, unregistered medical practitioners and chemists and that too in inadequate doses and duration to treat not only urinary tract infections but all kinds of infections. This may be the most important reason of high prevalence of ESBL producing organisms. Therefore to prevent emergence of resistance, efforts should be made to follow the indications for the administration of antibiotics and to use 
them appropriately for optimum period. Preferably antibiotics should be given on the basis of culture and sensitivity report. ${ }^{13}$ In this study $45.2 \%$ of isolate found to be ESBL producer by combination disk method which cannot detect ESBL when it is present in association with other $\beta$ lactamases like AmpC and MBL. ${ }^{1}$ The prevalence of ESBL producing strains reported from different geographical areas of our country among urinary isolates of E.coli was $58 \%$ in Delhi $^{14}, 41 \%$ in Coimbatore ${ }^{15}, 32 \%$ in Bijapur ${ }^{16}$, and $51 \%$ in Mangalore Karnataka. ${ }^{17}$ The variation in percentage of ESBL producing $E$. coli in different areas is probably due to the variation in the risk factors and extent of antibiotic use. Recent increase in number of ESBLs attributes to the emergence of CTX-M $\beta$-lactamase producing Enterobacteriaceae. ${ }^{18}$ Escherichia coli is the predominant CTX M group I ESBL producing member from Enterobacteriaceae. ${ }^{19}$

Among the aminoglycosides the resistance rate for Amikacin (12.5\%) and Gentamicin (40\%) was concordance with Singh et al. $2015(17 \%)^{20}$ and Dinesh kumar et al. $2014(32 \%)^{17}$ respectively. Among the fluoroquinolones, Ciprofloxacin (94\%) showed maximum resistance followed by norfloxacin $(90 \%)$ and levofloxacin $(88 \%)$. Such high level of resistance was also found in other studies in India. ${ }^{20}$ The possible explanation for the association between ESBL production and fluoroquinolones (Ciprofloxacin, Norfloxacin) resistance is the presence of genes of the two resistance mechanisms on the same plasmid. Besides this active efflux and outer membrane protein alterations are the other potential explanations. $^{22}$ Very high resistance in ESBL producers to Amoxycillin-clavulanic acid (75\%) were also found in near to Dinesh kumar et al. $2014(68 \%)^{21}$ and Hasan Ejaz et al. 2011(86\%) $)^{25}$, and more than that were found in Sasirekha et al. $2010(39 \%){ }^{23}$ Piperacillin shown resistance of $100 \%$ in our study, which is correspond to the R. Eshwar Singh et al. $2011^{24}$ and which is more than that of Dinesh kumar et al. $2014(80 \%)^{21}$, Sasirekha et al. $2010(83 \%)^{23}$, Hasan Ejaz et al.
2011(86\%). ${ }^{25}$ The resistance to Piperacillintazobactam $(27 \%)$ found is near to the Dinesh kumar et al. $2014(20 \%)^{21}$ and more than that were found in Sasirekha et al. $2010(9 \%)^{23}$ and Hasan Ejaz et al. 2011(10\% $)^{25}$. Trimethoprim- sulphmethoxazole has shown $83 \%$ resistance which is near to the Nair T Bhaskaran et al $2011(70 \%)^{26}$, Hasan Ejaz et al. 2011(78\%) ${ }^{25}$ and Singh et al. 2011 (78\%). $^{24}$ Nitrofurantoin showed 8\% resistance which is near to Nair T Bhaskaran et al $2011(9 \%)^{26}$ and less than that of Hasan Ejaz et al. $2011(28 \%)^{25}$, Singh et al. $2011(19 \%)^{24}$ and Dinesh kumar et al. $2014(42 \%)^{21}$. Tetracycline $(69 \%)$ resistance is near to Sasirekha et al. 2010 $(66 \%)^{23}$ Cefipime shown $94 \%$ resistance which is near to R. Eshwar Singh et al. $2011(94 \%)^{24}$ and more than to Dinesh kumar et al. $2014(42 \%){ }^{21}$ Carbapenems [Imipenem (2\%), Meropenem (4\%)] were shown lowest resistance which is higher than that were also found in Singh et al. $2015(0 \%)^{20}$, Sasirekha et al. $2010(0 \%)^{23}$ and Hasan Ejaz et al. 2011(1.3\%). ${ }^{25}$

\section{Conclusion}

Imipenem followed by Nitrofurantoin and Amikacin remains the drug of choice for ESBL producers with the highest sensitivity depending on severity and risk factors. In addition, to that the ESBL producing isolates were shown more resistance to many drugs which is again an alarming problem need to be monitored in routine at the earliest.

\section{References}

1. Khodare A, Mutha A, Purohit M. Prevalence of extended-spectrum $\beta$ lactamases producing Escherichia coli in urinary specimens and their phenotypic detection by modified three-dimensional enzyme extract tesh: Comparison with the Phenotypic confirmatory disc diffusion test. Indian J Microbiol Res 2017;4(3):244-247. 
2. Foman B. Epidemiology of urinary tract infections: Incidence, morbidity, and economic costs. Dis Mon 2003;49:53-70.

3. Stamm WE. Scientific and clinical challenges in the management of urinary tract infections. Am J Med 2002; 113:1S$4 \mathrm{~S}$.

4. Weinstein MP, Towns ML, Quartey SM, Mirrett S, Reimer LG, Parmigiani G, Reller LB. The clinical significance of positive blood cultures in the 1990s: a prospective comprehensive evaluation of the Microbiology, epidemiology, and outcome of bacteremia and fungemia in adults. Clin Infect Dis 1997; 24:584-602.

5. Pfaller MA, Jones RN. MYSTIC (Meropenem Yearly Susceptibility Test Information Collection) results from the Americas: resistance implications in the treatment of serious infections. $\mathrm{J}$ Antimicrob Chemother; (2000) 46 (Suppl):25-37.

6. Goossens H. (MYSTIC) Meropenem Yearly Susceptibility Test Information Collection) results from Europe: comparison of antibiotic susceptibilities between countries and centre types. J Antimicrob Chemother; (2000).(46 (suppl):39-52.

7. Monica Österblad, Antti Hakanen, Raija Manninen, Tiina Leistevuo, Reijo Peltonen, Olli Meurman et al. A between species comparison of antimicrobial resistance in enterobacteria in fecal flora. $\mathbf{J}$ Antimicrob Agents Chemother. 2000 Jun; 44(6): 1479-1484.

8. CLSI. Performance Standards for Antimicrobial Susceptibility Testing, Twenty fourth Informational Supplement, CLSI Document M100-S24, Wayne, PA: Clinical and Laboratory Standards Institute, 2014 .

9. Nathisuwan S, Burgess DS, Lewis II JS. ESBLs : Epidemiology, Detection and
Treatment. Pharmacotherapy 2001;21(8): 920-928.

10. Jacoby GA, Sutton L. (1991) Properties of plasmids responsible for production of extendedspectrum

$\beta$-lactamases. Antimicrob Agents Chemother 35: 164-69

11. Chessbrough, M 2006, District laboratory practice in tropical countries, Part-2, Newyork, USA: Cambridge university. pp. 105-115.

12. Hsueh PR, Hoban DJ, Carmeli Y, Chen $\mathrm{SY}$, Desikan $\mathrm{S}$, Alejandria $\mathrm{M}$ et al. Consensus review of the epidemiology and appropriate antimicrobial therapy of complicated urinary tract infections in Asia-Pacific region. J. Infect. 2011 Aug; 63(2): 114-23.

13. Ravinder Pal Singh, Sonali Jain, Amit Kumar Singh, Nikunj Gupta,. Dinesh Kumar. 1 and Hitesh Bhatt. Comparative study of ESBL producing Escherichia coli in OPD and IPD patients of urinary tract infections. Int.J.Curr.Microbiol.App.Sci (2013) 2(12): 45-50

14. Bedenic, B., N. Beader, and Z. Zagar. 2001. Effect of inoculum size on the antibacterial activity of cefpirome and cefepime against Klebsiella pneumonia strains producing SHV extended-spectrum beta-lactamases. Clin. Microbiol. Infect. 7:626-635.

15. Padmini S B, Raju B A, Mani K R. Detection of Enterobacteriaceae producing CTX-M extended spectrum $\beta$-lactamases from a tertiary care hospital in south India. Indian J Med Microbiol 2008;26:163-6

16. Metri Basavaraj C., Jyothi P., Peerapur Basavaraj V. The Prevalence of ESBL among Enterobacteriaceae in a Tertiary Care Hospital of North Karnataka, India. Journal of Clinical and Diagnostic Research. 2011 June, Vol-5(3): 470-475

17. Sharma S, Bhat GK, Shenoy S. Virulence factors and drug resistance in Escherichia coli isolated from extraintestinal 
infections. Indian J Med Microbiol. 2007

Oct; 25(4):369-73

18. Livermore DM, Hawkey PM. CTX M Changing The Face Of ESBLs in UK. J Antimicrob Chemother.2005;56:451-4.

19. Priyadharsini RI, Kavitha A, Rajan R, Mathavi S, Rajesh KR. Prevalence of bla ${ }_{\text {CтX }}$ Extended Spectrum Beta Lactamase Gene in Enterobacteriaceae from Critical Care Patients. J Lab Physicians. 2011 Jul-Dec; 3(2): 80-83

20. Singh S., Kumar S., Sandhu R., Devi $R$. and Yadav A.K.Prevalence of drug resistance in ESBL producing Escherichia coli causing UTI in rural tertiary care hospital from Haryana, India. International Journal of Basic and Applied Medical Sciences 2015 Vol. 5 (3) SeptemberDecember, pp. 1-7.

21. Kumar D, Singh AK, Ali MR, Chander Y. Antimicrobial Susceptibility Profile of Extended Spectrum $\beta$-Lactamase (ESBL) Producing Escherichia coli from Various Clinical Samples. Infectious Diseases: Research and Treatment 2014:7 1-8 doi:10.4137/IDRT.S13820

22. Livermoore DM and Paterson DL (2006). Pocket Guide to Extended Spectrum BLactamases in Resistance (New Delhi: Springer (India) Private Limited).

23. Sasirekha, B, Manasa, R, Ramya, P, and Sneha, R, 2010, Frequency and Antimicrobial Sensitivity Pattern Of Extended Spectrum $\quad \beta$-Lactamases Producing E.coli And Klebsiella Pneumoniae Isolated In A Tertiary Care Hospital, Al Ameen journal Medical science, vol.3, no.4, pp. 265-271.

24. R. Eshwar Singh, M. Veena, K.G. Raghukumar, G. Vishwanath, P.N. Sridhar Rao and B.V. Murlimanju. ESBL production: resistance pattern in Escherichia coli and Klebsiella pneumoniae, a study by DDST method.
International Journal of Applied Biology and Pharmaceutical Technology Volume: 2: Issue-4: Oct - Dec -2011

25. H Ejaz, K ul-Haq, A Zafa, S Mahmood, $M M$ Javed. Urinary tract infections caused by extended spectrum $\beta$-lactamase (ESBL) producing Escherichia coli and Klebsiella pneumonia. AJB Vol. 10(73), pp. 1666116666, 21 November, 2011

26. Nair T Bhaskaran, Bhat G Kishore, Pai Vidya, Shantharam Manjula. Extended spectrum $\beta$-lactamases (ESBL) in uropathogenic escherichia coli, prevalence and susceptibility pattern in a south indian city. IJRAP 2011, 2(6), 1756-1757. 